\title{
WNT signalling is both an inducer and effector of glucagon-like peptide-1
}

\author{
B. Gustafson • U. Smith
}

Received: 5 June 2008 / Accepted: 3 July 2008 / Published online: 30 July 2008

(C) Springer-Verlag 2008

Keywords Beta cells · $\beta$-Catenin - Insulin secretion · WNT signalling

\section{Abbreviations \\ GLP-1 glucagon-like peptide-1 \\ GSK-3 $\beta$ glycogen synthase kinase-3 $\beta$ \\ LRP LDL receptor-related protein \\ TCF T cell factor}

In this issue of Diabetologia, T. Jin [1] reviews our current understanding of how WNT signalling can be related to type 2 diabetes. The great interest in this topic stems from the pivotal finding by the Diabetes Epidemiology: Collaborative Analysis of Diagnostic Criteria in Europe (DECODE) organisation in Iceland [2] that polymorphisms in the TCF7L2 gene, which encodes a key nuclear receptor in the WNT signalling cascade, are strongly associated with the risk of type 2 diabetes. This finding has subsequently been reproduced in virtually all populations studied, and the TCF7L2 risk genotypes remain the most prominent genetic risks for type 2 diabetes.

No wonder many investigators have tried to unravel the molecular consequences of the TCF $2 L 2$ genotypes and, thus, components of the WNT signalling cascade. Under-

B. Gustafson · U. Smith $(\bowtie)$

The Lundberg Laboratory for Diabetes Research,

Center of Excellence for Metabolic and Cardiovascular Research,

Department of Molecular and Clinical Medicine,

The Sahlgrenska Academy at the University of Gothenburg,

SE-413 45 Gothenburg, Sweden

e-mail: ulf.smith@medic.gu.se standing the mechanisms involved may lead to new therapeutic interventions. In general, studies in which individuals carrying the risk genotypes have been carefully phenotyped mostly implicate reduced insulin secretion rather than increased insulin resistance [3, 4]. Thus, understanding how the WNT pathway influences beta cell number, differentiation and/or function is of major interest. Such information may also be important for type 2 diabetes at large, in particular, the loss of beta cell function with time.

\section{WNT: an ancient and ubiquitous signalling pathway}

WNT signalling is an extremely complex process which has to be very carefully controlled, both temporally and spatially. It is of fundamental importance for normal cell differentiation, cell-cell communication, organ formation and growth. This is underscored by the fact that it is highly conserved during evolution and probably evolved about 650 million years ago.

WNT proteins are cell-secreted glycoprotein ligands. They contain a conserved domain of approximately 300 amino acids, and this is punctuated by 21 to 23 cysteine residues with a characteristic spacing pattern. They are poorly soluble in water owing to a lipid (palmitoyl) chain attached to a conserved cysteine residue. Many cells, particularly undifferentiated or inflammatory cells, secrete WNT proteins. WNTs have a concentration-dependent effect; they are transported across large distances and can act on cells situated far from where they were originally produced. WNT activation is achieved through both paracrine and autocrine signalling. 
During stem cell regeneration, the WNT negative regulator Dickkopf-1 (DKK-1) allows stem cells to re-enter the cell cycle after the stationary phase. Induction of this regulator is an important mechanism that allows cells to limit WNT signalling and thereby prevent uncontrolled proliferation. In mesenchymal stem cells, WNT signalling regulates a reciprocal relationship between adipogenesis and osteogenesis.

Canonical WNT signalling is activated by the bridging of the Frizzled receptors and LDL receptor-related proteins (LRPs) by a secreted WNT protein, which elicits a signal that is transduced to several intracellular proteins, including axin, glycogen synthase kinase- $3 \beta$ (GSK- $3 \beta$ ) and Dishevelled (Fig. 1). Axin is important as it serves as a scaffold protein and has a rate-limiting function in the transduction of the WNT signal. Upon activation, axin binds to the intracellular region of the LRP receptor and is then degraded. GSK- $3 \beta$ becomes inactivated and intracellular $\beta$-catenin is stabilised and translocated to the nucleus,

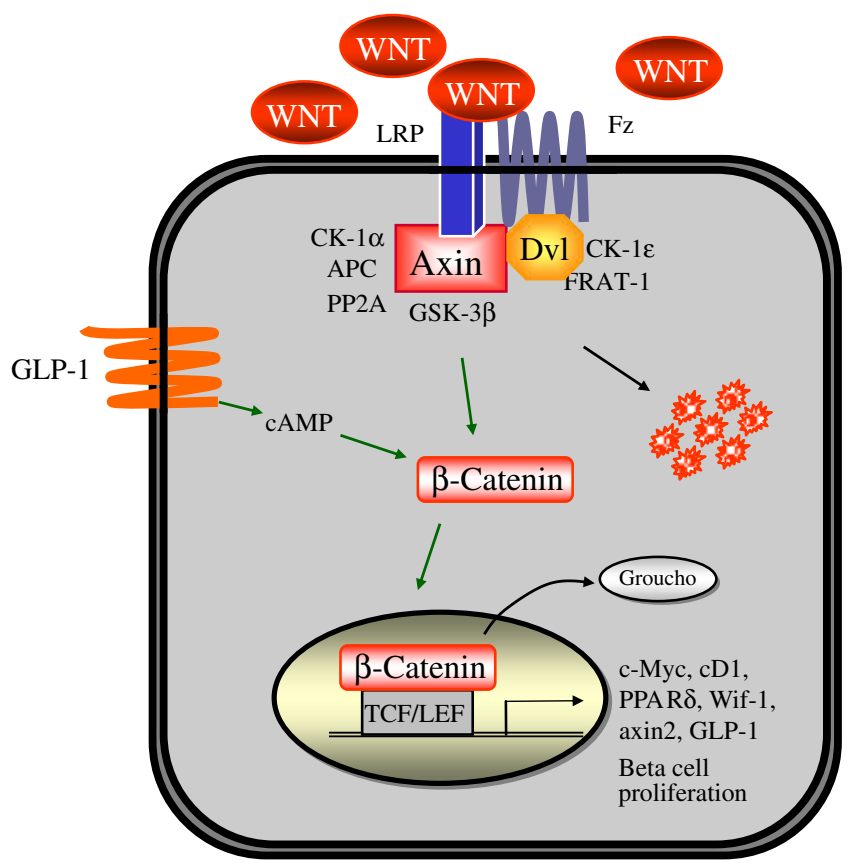

Fig. 1 When WNT molecules bind the frizzled (Fz) and LRP receptors, $\beta$-catenin levels are raised and bind to the nuclear TCF/ LEF (or TCF7L2) receptors after the dissociation of the repressor Groucho. Some of the genes activated, including that encoding GLP1 , are shown, as well as the ability of GLP-1, through its receptor and intracellular cAMP, to stabilise (increase) $\beta$-catenin and enhance beta cell proliferation. The red stars represent normal phosphorylated $\beta$-catenin targeted for destruction in the absence of activated WNT signalling. APC, adenomatous polyposis coli; CK, casein kinase; $\mathrm{cD}$, cyclin D1; DV1, Dishevelled; FRAT-1, frequently rearranged in advanced $\mathrm{T}$ cell lymphomas 1; LEF, lymphoid enhancer-binding factor; PP2A, protein phosphatase $2 \mathrm{~A}$; PPAR $\delta$, peroxisome proliferator-activated receptor $\delta$ where it replaces repressors and activates one of the members of the $\mathrm{T}$ cell factor (TCF) family (TCF7L2). Thus, $\beta$-catenin is the intracellular messenger in the canonical WNT signalling cascade and TCF7L2 the nuclear receptor, which, in turn, activates the transcription of WNTregulated genes.

\section{TCF7L2 and type 2 diabetes}

Most, but not all, studies have found insulin secretion to be reduced in carriers of the TCF $7 L 2$ risk genotypes. In his review, Jin [1] discusses potential mechanisms relating WNT signalling (and $\beta$-catenin) to pancreatic development and beta cell function, as well as the involvement of this signalling pathway in incretin regulation, in particular, glucagon-like peptide-1 (GLP-1) secretion and/or effects.

The majority of studies support the concept that a wellfunctioning WNT signalling pathway is necessary for the normal development and function of beta cells. One specific area that has received much attention is the possibility that the TCF7L2 risk genotypes are associated with impaired GLP-1 secretion and/or effect. The work by Jin and co-workers [5] showing that the GLP-1-producing $\mathrm{L}$ cells are dependent on $\beta$-catenin for GLP-1 secretion suggested GLP-1 as the link between the TCF7L2 risk genotypes and type 2 diabetes. However, several large studies did not show reduced GLP-1 levels in individuals carrying the risk genotypes [3, 4]. Instead, it was suggested that the effect of GLP-1 was reduced, and, indeed, infusion of GLP-1 in risk genotype carriers produced a lower insulin response [3, 4]. However, evidence from Holst and coworkers [6] that even minor increases in postprandial glucose levels reduce the incretin effects, makes this conclusion conjectural. The reduced GLP-1 effect may therefore be secondary to higher postprandial glucose levels in carriers of a $T C F 7 L 2$ risk genotype. These findings raise serious concerns about the role of GLP-1, both in terms of its own secretion and its effect on insulin secretion as a mechanism for the association of TCF7L2 genotypes with type 2 diabetes. However, Liu and Habener [7] recently reported that TCF7L2 and $\beta$-catenin are important for the stimulating effect of GLP-1 on beta cell proliferation. These authors clearly show that GLP-1, via the GLP-1 receptor, activates WNT signalling in both pancreatic beta cells and the INS- 1 cell line. This occurs through the activation of the cAMP-dependent protein kinase A. This protein, in turn, phosphorylates Ser-675 of $\beta$-catenin, stabilising it so that it can enter the nucleus, leading to the nuclear activation of transcription through TCF7L2. These authors also show that active WNT signalling is required for the proliferation of INS-1 cells, both in the non-stimulated and the GLP1- 
stimulated states [7]. In this scenario, the impaired insulin secretion in the TCF7L2 risk genotypes would be associated with a reduced beta cell mass as a long-term consequence, rather than as an incretin effect on insulin secretion. Unfortunately, addressing this in man is currently not feasible since we lack reproducible methods of determining beta cell mass.

In conclusion, WNT signalling is now emerging as a key pathway for beta cell growth and differentiation. The recent findings linking GLP-1 as a direct activator of this pathway and beta cell growth (Fig. 1) are highly exciting and may have ramifications not only for the $T C F 7 L 2$ risk genotypes, but also for the progressive reduction of beta cell mass in type 2 diabetes.

Duality of interest The authors declare that there is no duality of interest associated with this manuscript.

\section{References}

1. Jin $\mathrm{T}$ (2008) Wnt signalling pathway and diabetes mellitus. Diabetologia doi:10.1007/s00125-008-1084-y

2. Grant SF, Thorleifsson G, Reynisdottir I et al (2006) Variant of transcription factor 7-like 2 (TCF7L2) gene confers risk of type 2 diabetes. Nat Genet 38:320-323

3. Lyssenko V, Lupi R, Marchetti P et al (2007) Mechanisms by which common variants in the TCF7L2 gene increase risk of type 2 diabetes. J Clin Invest 117:2155-2163

4. Schafer SA, Tschritter O, Machicao F et al (2007) Impaired glucagon-like peptide-1-induced insulin secretion in carriers of transcription factor 7-like 2 (TCF7L2) gene polymorphisms. Diabetologia 50:2443-2450

5. Ni Z, Anini Y, Fang X, Mills G, Brubaker PL, Jin T (2003) Transcriptional activation of the proglucagon gene by lithium and betacatenin in intestinal endocrine L cells. J Biol Chem 278:1380-1387

6. Knop FK, Vilsboll T, Hojberg PV et al (2007) Reduced incretin effect in type 2 diabetes: cause or consequence of the diabetic state? Diabetes 56:1951-1959

7. Liu Z, Habener JF (2008) Glucagon-like peptide-1 activation of TCF7L2-dependent Wnt signaling enhances pancreatic beta cell proliferation. J Biol Chem 283:8723-8735 